\title{
CULTURA DE USO Y EXPLOTACIÓN ECONÓMICA DE LAS PLAYAS EN CARTAGENA DE INDIAS
}

\author{
MARTHA YÁNEZ CONTRERAS* \\ ALBERTO ORDOÑEZ FLÓREZ \\ LUIS SUÁREZ ÁVILA ${ }^{* * *}$
}

Recibido 21 de Octubre de 2009/Enviado para Modificación 18 de Noviembre de 2009/Aceptado 30 de Noviembre de 2009

\section{RESUMEN}

El objetivo del presente artículo es mostrar la caracterización en términos socioeconómicos de la población de trabajadores estacionarios informales ubicados en las playas de Cartagena de indias, y determinar la influencia de elementos de percepción institucional y cultural como el reconocimiento de las autoridades competentes y el desarrollo de mecanismo de pseudo propiedad sobre las prácticas de explotación económica de este espacio. Para ello se utilizan los resultados de una encuesta aplicada a los vendedores estacionarios informales, ubicados en las playas de los sectores de Bocagrande y Laguito en Cartagena. Efectuando un diseño por cuotas se entrevistaron 85 vendedores estacionarios, distribuidos en 25 vendedoras de frutas, 30 carperos y 30 vendedores en módulos. A estos se les aplicó, como parte del trabajo de recolección de información, un formulario con una estructura que indagaba sobre aspectos económicos (ingresos, inversión, precios), sociales (educación, salud), culturales (concepciones e ideas respecto al puesto de trabajo), generales (jornada laboral) y de control institucional (carnetización, uniforme). La información recolectada se procesó con el programa estadístico SPSS 15.0, para generar los cuadros de salida y relacionar las variables de interés. Se desarrolló por último un estudio de la asociación entre variables por medio de análisis de contingencia. Los resultados obtenidos muestran que la demanda inadecuada d las playas es utilizada por los vendedores como una estrategia para aumentar sus ingresos. Por otra parte se observa el desarrollo de mecanismos de privatización de este espacio por parte de los vendedores con mayor número de años explotándolo económicamente, quienes utilizan los instrumentos de regulación institucional para crear barreras a la entrada de nuevos competidores en las zona de su interés.

\footnotetext{
* Jefe Departamento de Investigaciones Económicas y Sociales (DIES), Universidad de Cartagena. Correo Electrónico: iceconomicas@unicartagena.edu.co

** Economista Universidad de Cartagena. Especialista en Gestión Gerencial Universidad Tecnológica de Bolívar. Correo Electrónico: aordonez@repcaltda.com.

*** Economista Universidad de Cartagena. Correo Electrónico: lucho0227@hotmail.com.
} 
Palabras Clave: Espacio público, Playas, vendedores informales, explotación económica

Clasificación Jel: H41, R12, Q21

\begin{abstract}
The aim of the present article is to show the socioeconomics characterization of the population of stationary informal workers located in the Cartagena's beaches and to determinate the influence of elements of institutional and cultural perception as the recognition of the competent authorities and the development of mechanism of pseudo property on the practices of economic exploitations of this area. For that are used the results of a survey applied to the stationary informal seller, located in the beaches of Bocagrande and Laguito in Cartagena. Doing a design by quota, it was interviewed 85 stationary sellers, distributed in 25 fruits saleswoman, 30 carpers and 30 sellers in modules. For this sellers it was applied, as part of the information collection work, a form with a structure that wonders about economic aspects (income, invest, price), socials (education, health), culturals (conceptions and ideas about the job), generals (working day), and of institutional control (carnetization, uniform). The data was processed with the software SPSS 15.0, to generate the input tables and to relate the variables of interest. Finally, it was developed a study of the association between variables through the contingency analysis. The results show that the inadequate demand of the beaches is used by the seller as a strategy to increase their incomes. On the other hand, it is observed the development of privatization mechanisms of this area by the sellers with the major quantity of years exploiting it economically, who uses the instruments of institutional regulations to create entrance barriers of new competitors in the interest zones.
\end{abstract}

Key Words: Espacio público, Playas, vendedores informales, explotación económica

JEL Classification: H41, R12, Q21

\title{
INTRODUCCIÓN
}

Las playas de Cartagena son uno de los principales atractivos turísticos de esta ciudad, situada al norte de Colombia a orillas del Mar Caribe. La gran afluencia de turistas nacionales y extranjeros, quienes las visitan permanentemente para disfrutar de sus atributos, atrae la presencia 
de un grupo importante de individuos del sector formal e informal de la economía, quienes ofrecen sus productos y servicios a propios y visitantes, convirtiéndola en una importante fuente de empleo local.

Al principio, las ventas informales en las playas se declararon ilegales ${ }^{1}$, pero progresivamente se fueron aceptando con la finalidad de ofrecer servicios más diversos a los visitantes. Esta aceptación se vio materializada en la construcción de módulos que buscaban organizar a las personas que de forma ambulante vendían sus productos en la playa. Esta estrategia mostró inicialmente resultados positivos; sin embargo, en la actualidad las playas se encuentran generalmente saturadas de vendedores, cuyo acoso permanente causa molestias a los turistas y externalidades negativas sobre su entorno próximo (2). La tendencia creciente de la demanda por usos de las playas de Cartagena, ha carecido de un control eficiente y continuo, dando lugar a la ocupación formal e ilegal de espacios de gran diversidad biológica y riqueza paisajística. En consecuencia, este espacio se ha convertido en un territorio en disputa entre quienes lo demandan con fines de explotación económica y quienes lo hacen con fines recreativos. Hoteles, restaurantes y comerciantes que desde la formalidad conviven y hasta compiten con vendedores ambulantes y estacionarios informales, que han encontrado en este sitio un mercado propicio para una oferta diversa de bienes y servicios, cuya venta les provee de un ingreso que les ha negado la baja capacidad de absorción del mercado laboral local. En el otro lado, los usuarios con fines recreativos, ven reducidos los lugares de esparcimiento que les permitan disfrutar tranquilamente de los atributos naturales, los espacios de socialización y encuentro que ofrecen las playas.

Por otra parte, la ausencia de control sobre su explotación económica ha dado paso al desarrollo de una cultura de ausencia de reconocimiento de la autoridad encargada de su ordenamiento y al desarrollo de "conciencias de propiedad" de los vendedores sobre el espacio público que explotan.

El fenómeno ha crecido en tales proporciones que, cada vez con más frecuencia, el tema de organización y control de las ventas ambulantes, hace parte del diseño de estrategias de recuperación de playas. Prueba de ello es su

${ }^{1}$ El decreto 328 del 7 de abril de 1995 (1), expedido por la Alcaldía Mayor de Cartagena de Indias, estableció la prohibición de ventas ambulantes y estacionarias en los sectores turísticos del distrito de Cartagena, en especial los barrios de Bocagrande, Laguito, Castillogrande, Crespo y Marbella, los corregimientos y veredas con zonas costeras o de playas, a fin de frenar la proliferación de estas ventas y garantizar a los habitantes y visitantes los derechos al uso y goce pacifico y tranquilo de las zonas y espacios públicos. 
inclusión como elemento clave para el desarrollo del sector turístico local y nacional, en los Planes sectoriales de Colombia de los periodos 2003-2006 (3) y 2007-2010 (4). A nivel local el Plan Sectorial de Turismo de Cartagena de Indias (5), hace énfasis en la estrategia de "recuperación, adecuación y reglamentación de las playas del distrito...para que sean explotadas correctamente, se genere empleo formal y sean competitivas internacionalmente".

Teniendo en cuenta que el empleo de mecanismos de choque o desalojo forzoso tendría un gran impacto social sobre las personas que derivan su ingreso principal de estas actividades económicas, se han considerado mecanismos de cooperación alternativos como los procesos de reubicación concertada. Cualquiera que sea la estrategia escogida para alcanzar este objetivo debe partir de considerar las características que distinguen la población afectada y el entorno cultural que potenciaría o dificultaría su éxito.

El presente estudio, utiliza los resultados de una encuesta aplicada a los vendedores estacionarios informales, ubicados en las playas de los sectores de Bocagrande y Laguito en Cartagena. A partir de ella se realiza una caracterización en términos socioeconómicos de la población de trabajadores estacionarios informales, y se determina la influencia de elementos de percepción institucional y cultural como el reconocimiento de las autoridades competentes y el desarrollo de mecanismo de pseudo propiedad del espacio público explotado económicamente, sobre las decisiones de ubicación de los vendedores en las playas.

El documento se organiza en ocho partes, que inician con esta introducción. En la segunda se muestra el marco teórico y empírico que soporta en la formulación y el desarrollo del trabajo. La descripción del proceso metodológico se tiene en la siguiente sección. Los elementos de tipo jurídico y legal que atañen al espacio público y en especial a las playas se sintetiza en la sección cuarta; el apartado quinto explica la forma en que la Dirección General Marítima (DIMAR), autoridad en lo referente a las playas, zonifica y asigna el espacio disponible. La sección seis contiene los resultados y comentarios, productos del sondeo efectuado a los vendedores estacionarios. Finalmente, la sección séptima concluye y muestra recomendaciones y la octava referencia la bibliografía empleada.

\section{MARCO REFERENCIAL}

Los bienes públicos se caracterizan por ser no rivales y no excluyentes en su consumo. La no rivalidad de un bien, se refiere a que el consumo de una persona no impide o reduce la cantidad disponible de este, para 
consumo de otra. La no exclusión alude a la imposibilidad de negar el acceso o consumo del bien a cualquier persona. La escasez de ejemplos de bienes que cumplan al tiempo con estas dos propiedades, ha llevado a clasificarlos con el rotulo distintivo de bienes públicos puros.

En general, las playas de uso recreacional son un tipo especial de bien público, llamado bien común. Aunque el consumo de los atributos de este recurso se presenta bajo condiciones de no exclusión, se torna rival a niveles crecientes de su utilización; dando lugar a problemas de congestión eventual (6). De esta forma, si la demanda por el espacio de las playas es baja (bajo número de visitantes y comerciantes), estas se comportan como un bien no rival. Aunque un demandante adicional de las playas obtiene un beneficio positivo de su uso o explotación, impone un costo marginal al resto de usuarios, igual a cero. Desde el punto de vista económico, en esta situación, cualquier mecanismo de exclusión resulta ineficiente ya que el consumo adicional de las playas no tiene costo marginal, y un mecanismo de exclusión solo provocaría subconsumo (7).

Sin embargo, dado que las playas tienen una extensión fija, una vez alcanzado el límite de su capacidad, el recurso se vuelve rival y aparece la congestión. A partir de allí, el uso de la playa por una persona adicional afecta el uso o explotación de ella por el resto de usuarios, provocando costos marginales sociales positivos. Normalmente, la decisión del individuo acerca de usar o no un recurso común y en qué cantidad, solo tendrá en cuenta los costos y beneficios que lo afectan directamente. El consumo de un recurso rival bajo condiciones de no exclusión incentiva a los consumidores para capturar la mayoría de los beneficios que provee el activo antes que otra persona los capture primero. En este caso la ineficiencia económica se presenta por sobre-uso del recurso (8). De acuerdo a Hardin (9), la solución está en dejar de tratar a los recursos naturales como recursos comunes. El autor plantea dos opciones: venderlos como propiedad privada o mantenerlos como propiedad pública acompañado de algún mecanismo de exclusión, que impida la superación del límite de su capacidad.

La opción de venta como propiedad privada encuentra restricciones de tipo moral e institucional, este último representado en el marco legal de los bienes públicos en Colombia.

La Constitución Política de Colombia de 1991 (10), en su artículo 82 reconoce que "Es deber del Estado velar por la protección de la integridad del espacio público y por su destinación al uso común, el cual prevalece sobre el interés particular". Adicionalmente, el Artículo 679 del Código 
Civil (11) resalta que "las playas marítimas son bienes de uso público", los cuales conforme al artículo 63 de la constitución de 1991 (10) son de carácter "inembargable, inalienable e imprescriptible".

Alcaldes y Concejos distritales son llamados a defender y recuperar el espacio público, mediante la provisión 7 del decreto 1421 de 1993, al responsabilizarlos de: "Dictar los actos y ejecutar las operaciones necesarias para la protección, recuperación y conservación del espacio público, el patrimonio cultural, arquitectónico e histórico, los monumentos de la localidad, los recursos naturales y el ambiente, con sujeción a la ley, a las normas nacionales aplicables, y a los acuerdos distritales y locales". La imposibilidad de asignar precios de acceso a la playa y dejar al mecanismo de mercado la corrección de las ineficiencias crea situaciones de suboferta en el espacio público y recrea las condiciones para que el Estado sea responsabilizado de suministrarlo.

En este marco legal, el Estado participa como agente del colectivo social. A nivel local, los mecanismos escogidos para asumir este papel han sido han sido variados. En 1995 el decreto 328 (1) de la alcaldía de Cartagena, estableció “La prohibición de ventas ambulantes y estacionarias en los sectores turísticos del distrito, en especial los barrios de Bocagrande, Laguito, Castillogrande, Crespo y Marbella, los corregimientos y veredas con zonas costeras o de playas, debido a la proliferación de estas ventas". A fin de "garantizar a los habitantes y visitantes los derechos al uso y goce pacifico y tranquilo de las zonas y espacios públicos". Sin embargo, años más tarde, esta posición se flexibilizó y en enero del año 2000, la Alcaldía Mayor de Cartagena y la DIMAR expiden la reglamentacion para vendedores de módulos, carperos, silleteros y vendedores de frutas en las playas del distrito.

Aunque la Ley 768 del 31 de julio de 2002 (12) transfirió a los consejos distritales la responsabilidad de expedir las normas con base en las cuales se reglamentaran las actividades turísticas, recreacionales, deportivas en las playas y demás espacios de uso público, exceptuando las zonas de bajamar. Sin embargo, en 2006 dicha ley no se había reglamentado en Cartagena, por lo que todo lo relacionado con el uso del espacio público en las playas de la ciudad seguía reglamentándose principalmente por la Resolución 438 de 1986 (13) y el Decreto-Ley 2324 de 1984 (14), emitidos por la Dirección General Marítima (DIMAR), apoyadas por las resoluciones emitidas por la Alcaldía Distrital sobre dicha materia.

La ambigüedad de marco legal, la flexibilidad del marco regulatorio y la ineficiencia en la ejecución de políticas de control han convertido las 
playas cartageneras en un ejemplo de recurso natural apropiado como bien común, pero explotado como competencia individual (15). En un escenario competitivo grupos de vendedores de productos similares buscan diferenciarse de sus competidores a partir de la elección de su ubicación. Mientras más cerca se encuentren de sus clientes, menor será la molestia que soportará un turista por transportar sus compras hasta el lugar que ocupan en la zona de descanso. Las preferencias de los compradores se inclinarán por el establecimiento o vendedor que le represente menores molestias de desplazamiento y transporte de las mercancías compradas. Se crean así incentivos para que los vendedores abandonen las zonas de trabajo inicialmente asignadas y tiendan a concentrarse en los espacios de mayor afluencia de potenciales compradores o invadir aquellos reservados para tránsito o descanso de los turistas. El vendedor más alejado intentará ubicarse cerca de aquel que tiene mejor localización e incrementar su ganancia a expensas de la de este (16). A medida que un mayor número de ellos tienden a concentrarse en el mismo lugar, se presentan externalidades negativas de aglomeración para vendedores, representadas por disminución de sus ganancias individuales y de congestión para todos los usuarios.

De esta forma, cuando para un vendedor en la playa, el problema económico a resolver no es cuánto producir sino donde operar, la decisión de distribuirse asimétricamente sobre esta le permite conservar las ventajas de ubicación que la aglomeración elimina. La aplicación de la teoría de juegos a la explotación de los recursos comunes ha sido explorada con particular interés en la soluciones de tipo cooperativo ${ }^{2}$. Sin embargo, los altos costos de información y transacción que involucran los acuerdos, así como la dificultad de establecer sistemas de monitoreo y sanción para los violadores, ha limitado el éxito de estas estrategias. En materia de espacio público, las soluciones concertadas normalmente se traducen en acuerdos de reubicación o compensación.

Desde la literatura nacional e internacional, el tema ha sido explorado con regularidad, y ha predominado el tratamiento del fenómeno de crecimiento de los vendedores ambulantes y su invasión de otros espacios como las calles y parques urbanos. Donovan (18) en un trabajo muy completo, describe la evolución de los obstáculos legales, políticos y económicos que ha enfrentado el proceso de recuperación de espacio público en Bogotá.

Vázquez Ramírez (19), en un análisis de la invasión de las plazas y calles de Pereira, afirma:

${ }^{2}$ Para varios ejemplos mirar Ostrom (17). 
" Cuando la racionalización en el uso del espacio público se tiene que hacer en términos de defensa, quedan en evidencia dos cosas: la primera, quienes lo usan lo invaden, es decir, lo ocupan sin ninguna reglamentación, pero además usan en beneficio particular algo que pertenece a la Res pública y que va en detrimento del bienestar común; la segunda es la constatación de una economía sumergida que crece paralela a la desigualdad social y que al desbordarse en el ámbito público, sólo encuentra como solución acciones de control".

Rocha y Sánchez (20), ensayan una valoración económica de las externalidades atribuibles a la presencia de vendedores ambulantes en el espacio público de Bogotá. A partir de un censo aplicado a establecimientos de comercio y ventas callejeras en cuatro importantes sectores comerciales de la ciudad, los autores construyen un modelo de corte transversal para calcular el efecto de la presencia de ventas callejeras sobre las ventas y empleo de los establecimientos comerciales. A partir de estos resultados se hicieron simulaciones de impacto de la aplicación de políticas de reubicación sobre el empleo e ingresos de comerciantes y vendedores. Al final se concluye acerca del bajo potencial de la explotación económica del espacio público en términos de generación de valor agregado, empleo e impuestos

\section{METODOLOGÍA}

Con el fin de identificar las características de los vendedores estacionarios de las playas de Cartagena de Indias, se procedió a delimitar geográficamente el estudio a la zona de Bocagrande y El Laguito, debido a que son escenarios de amplia concurrencia de prestadores de servicios afines a las playas, específicamente fueron de interés los proveedores de tres tipos de bienes: a) carperos (aquellos que ofrecen en modo de alquiler carpas, sombrillas, sillas o esteras a los visitantes de las playas), b) vendedores de frutas y c) vendedores de productos y servicios varios ubicados un lugar fijo a modo de cabina o local. El trabajo de campo tuvo lugar entre los meses de julio de 2004 y febrero de 2005.

Para el cálculo de la muestra se tomaron los registros disponibles en la DIMAR, hasta 2002, que contabilizaban una población total $(N)$ de 235 vendedores (28,5\% fruteras, 30,2\% carperos y 42,1\% vendedores en módulos). 
Teniendo en cuenta que los anteriores datos de trabajadores informales estaban disponibles solo hasta 2002, se proyectó el tamaño de este grupo aplicando la tasa de crecimiento del trabajo informal para Cartagena, calculada por el DANE. En 2003 tal crecimiento fue de -3,55\% y en 2004 de 4,65\%; la población proyectada que se obtiene corresponde a 237 vendedores.

Se empleó la fórmula (1), operando con un error $i$ del 8,5\%, un nivel de confianza $\alpha$ del $95 \%$ (con un $Z$ asociado del 1,96) y niveles de prevalencia $p$ y $q(=p-1)$ desconocidos, correspondientes al $50 \%$.

$$
n=Z_{\alpha}^{2} \frac{N p q}{i^{2}(N-1)+Z_{\alpha}^{2} p q}
$$

Efectuando un diseño por cuotas se obtuvo una muestra $(\boldsymbol{n})$ de 85 vendedores estacionarios, distribuidos en 25 vendedoras de frutas, 30 carperos y 30 vendedores en módulos. A estos se les aplicó, como parte del trabajo de campo, un formulario de 56 preguntas con una estructura que indagaba sobre aspectos económicos (ingresos, inversión, precios), sociales (educación, salud), culturales (concepciones e ideas respecto al puesto de trabajo), generales (jornada laboral) y de control institucional (carnetización, uniforme).

La información recolectada se procesó con el programa estadístico SPSS 15.0, para generar los cuadros de salida y relacionar las variables de interés.

Se desarrolló en este documento un estudio de la asociación entre variables por medio de análisis de contingencia. En primer lugar se relacionaron algunas variables potencialmente explicativas de la demanda inadecuada de la zona de la playa en que se ubican los vendedores estacionarios, entendiéndose este mal uso como el encontrarse el vendedor fuera de los límites establecidos legalmente para el desarrollo de su negocio, que como más adelante se podrá ver, corresponde al área de reposo.

Las variables a considerar fueron el reconocimiento y la presencia de la autoridad, si el vendedor es reubicado $(1=\mathrm{S} 1$, $0=\mathrm{No})$, las intenciones de legar el negocio $(1=\mathrm{Sí}, 0=\mathrm{No})$, el tiempo que se tiene laborando en la playa, el género del individuo ( $1=$ Hombre, $0=$ Mujer $)$, la condición de ser pionero $(1=\mathrm{Sí}, 0=\mathrm{No})$ y el ingreso promedio mensual devengado por el desarrollo de la actividad en la playa.

El reconocimiento acertado de la autoridad, se refiere a la institución que el trabajador reporta reconocer como reguladora del espacio playero y corresponde a una variable dummy que asume el valor de 1 si manifestó 
ser la Alcaldía Mayor de Cartagena o la DIMAR. El tiempo de laborar en la playa se manejó con rangos, al igual que el ingreso promedio recibido al mes; para la primera variable se establecieron quinquenalmente, mientras que respecto a la última fueron tres rangos (\$100.000 a $\$ 300.000$, $\$ 300.000$ a $\$ 600.000$ y mayor a $\$ 600.000)$.

La segunda parte del análisis de contingencia está referido a caracterizar el tipo de prácticas, que los vendedores estacionarios, desarrollan en la playa según el tiempo de permanencia que tienen en ésta. Se considera como "buenas prácticas" el uso de algún mecanismo de identidad y reconocimiento en el trabajo (uniforme), la ubicación dentro de los límites establecidos para ejercer su labor (demanda adecuada del espacio público de la playa), el mantener aseado el lugar de trabajo, reconocer a la autoridad marítima (Alcaldía de Cartagena o DIMAR) y manifestar llevar cordiales relaciones con los vecinos.

En las tablas de contingencia que se elaboraron son útiles los siguientes estadísticos. El $X^{2}$ permite comprobar la hipótesis de la existencia de asociación entre variables, si resulta ser estadísticamente significativo $(<0,05)$ quiere decir que se descarta la independencia de la asociación. La V de Crámer y el coeficiente de contingencia miden el grado de asociación, entre más cercana a la unidad estén, mayor es el grado de relación entre las variables. Finalmente el coeficiente de incertidumbre permite determinar el grado de incertidumbre que se logra reducir en una predicción, luego de considerar que una variable es dependiente de otra.

\section{ZONIFICACIÓN DEL ESPACIO PÚBLICO EN LAS PLAYAS}

Teniendo en cuenta lo dispuesto en la Resolución 438 de 1986 (13), el espacio público en las playas del Distrito de Cartagena de Indias se organiza y zonifica de la siguiente forma:

Una a) zona netamente pública con una extensión de $50 \mathrm{mts}$ a partir de la línea de más alta marea, que se estructura en: i) área activa que es la franja de arena más próxima a la orilla del mar, la cual deberá tener un mínimo de $15 \mathrm{mts}$. permanecer libre en la totalidad de su longitud para favorecer la cómoda inmersión y la circulación de los bañistas; ii) área de reposo, la franja inmediata y paralela al área anterior y deberá tener un mínimo de 20 mts. en ésta se permite la colocación de sillas, esteras, toldos, parasoles carpas y otros elementos portátiles para hacer más cómoda la permanencia de los bañistas y turistas que visitan cada una de las playas; 
un iii) área de transición, la franja inmediata y paralela al área anterior, deberá tener un mínimo de $15 \mathrm{mts}$. en esta área se permite la práctica de deportes colectivos, en ésta en época alta de turismo se desarrollan campeonatos nacionales e internacionales deportivos. Vale anotar que en la zona netamente pública no se permitirá la construcción de ninguna infraestructura de carácter permanente.

Está igualmente la $b$ ) zona de concesión que es inmediata y paralela a la zona netamente pública, extendiéndose hasta el lugar donde se presente un marcado cambio en la forma fisiográfica, o hasta donde se inicia la línea de vegetación permanente, ésta podrá ser otorgada para uso y goce por la DIMAR, en esta área se permite la construcción de infraestructuras permanentes como kioscos y casetas salvavidas. A la Capitanía de Puerto, como autoridad marítima y ambiental, le corresponde la expedición de permisos para concesiones, instalación de sillas, toldos, carpas y venta de frutas con fines de explotación comercial y de prestación de servicios al turista en las áreas de reposo y transición. Para la delimitación cartográfica de las playas de Bocagrande y El Laguito (ver Cuadro 1 y Planos 1, 2a y 2b), se efectúa una división en tres sectores con sus respectivas coordenadas de ubicación, y luego se presenta el total del área de estudio en metros cuadrados.

CUADRO No. 1. DELIMITACIÓN CARTOGRÁFICA DE LAS PLAYAS OBJETO DE ESTUDIO

\begin{tabular}{|c|c|c|c|c|}
\hline \multirow[b]{2}{*}{ Coordenadas } & \multirow{2}{*}{$\begin{array}{l}\text { El Laguito } \\
75^{\circ} 34^{\prime} 15^{\prime \prime} \mathrm{W} \\
75^{\circ} 33^{\prime} 45^{\prime \prime} \mathrm{W} \\
10^{\circ} 23^{\prime} 55^{\prime \prime} \mathrm{N} \\
10^{\circ} 24^{\prime} 15^{\prime \prime} \mathrm{N}\end{array}$} & \multicolumn{2}{|l|}{ Bocagrande } & \multirow[b]{2}{*}{ Total } \\
\hline & & $\begin{array}{l}75^{\circ} 33^{\prime} 50^{\prime \prime} \mathrm{W} \\
75^{\circ} 33^{\prime} 20^{\prime \prime} \mathrm{W} \\
10^{\circ} 24^{\prime} 15^{\prime \prime} \mathrm{N} \\
10^{\circ} 24^{\prime} 35^{\prime \prime} \mathrm{N}\end{array}$ & $\begin{array}{l}75^{\circ} 33^{\prime} 35^{\prime \prime} \mathrm{W} \\
75^{\circ} 33^{\prime} 05^{\prime \prime} \mathrm{W} \\
10^{\circ} 24^{\prime} 30^{\prime \prime} \mathrm{N} \\
10^{\circ} 24^{\prime} 55^{\prime \prime} \mathrm{N}\end{array}$ & \\
\hline Zona Activa & $13.610 \mathrm{~m}^{2}$ & $14.360 \mathrm{~m}^{2}$ & $9.590 \mathrm{~m}^{2}$ & $37.560 \mathrm{~m}^{2}$ \\
\hline Zona de Reposo & $14.530 \mathrm{~m}^{2}$ & $18.150 \mathrm{~m}^{2}$ & $8.100 \mathrm{~m}^{2}$ & $40.780 \mathrm{~m}^{2}$ \\
\hline Zona de Transición & $10.480 \mathrm{~m}^{2}$ & $10.480 \mathrm{~m}^{2}$ & $1.670 \mathrm{~m}^{2}$ & $22.630 \mathrm{~m}^{2}$ \\
\hline Total & $38.620 \mathrm{~m}^{2}$ & $42.990 \mathrm{~m}^{2}$ & $19.360 \mathrm{~m}^{2}$ & $100.970 \mathrm{~m}^{2}$ \\
\hline
\end{tabular}

Fuente: Diseño de los autores con base en Gutiérrez (21)

De acuerdo a lo presentado en el Cuadro No. 1, si se tiene en cuenta el supuesto de que una persona ocupa un área física de $1 \mathrm{~m}^{2}$, se establece entonces que las playas turísticas de El Laguito y Bocagrande pueden soportar un máximo de 100.970 personas en las zonas identificadas; 37.560 personas en tránsito en la zona activa; 40.780 personas ubicadas en las carpas instaladas en la zona de reposo y 22.630 personas repartidas en la zona de transición y práctica de deportes de playeros. 
PLANO No. 1. PLAYA SECTOR EL LAGUITO

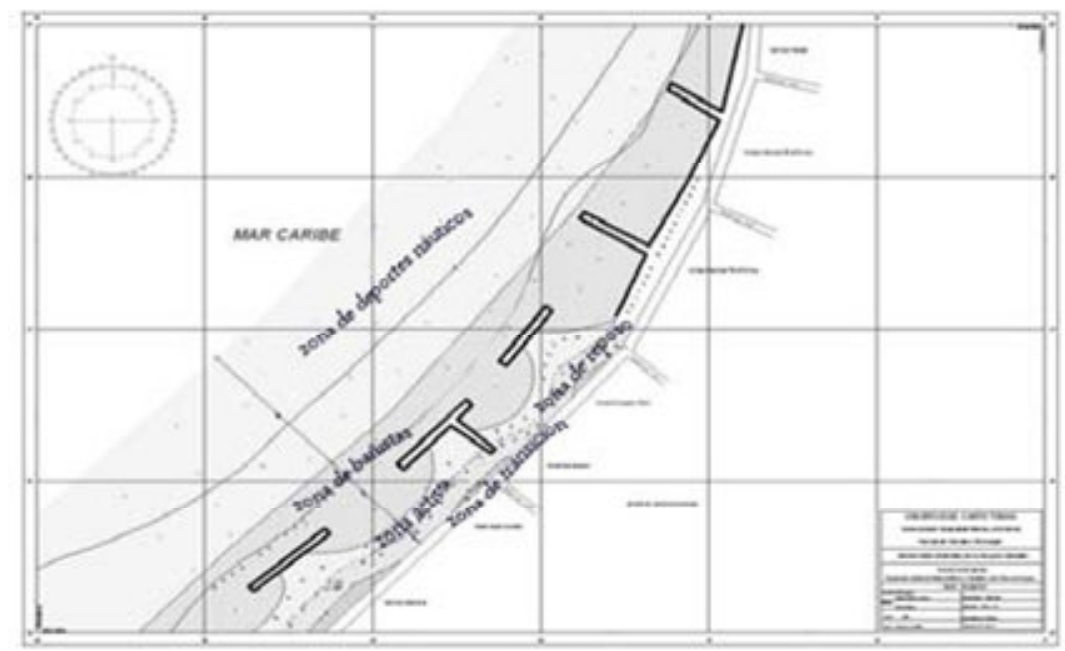

Fuente: Gutiérrez (21)

PLANO No. 2a. PLAYA SECTOR BOCAGRANDE

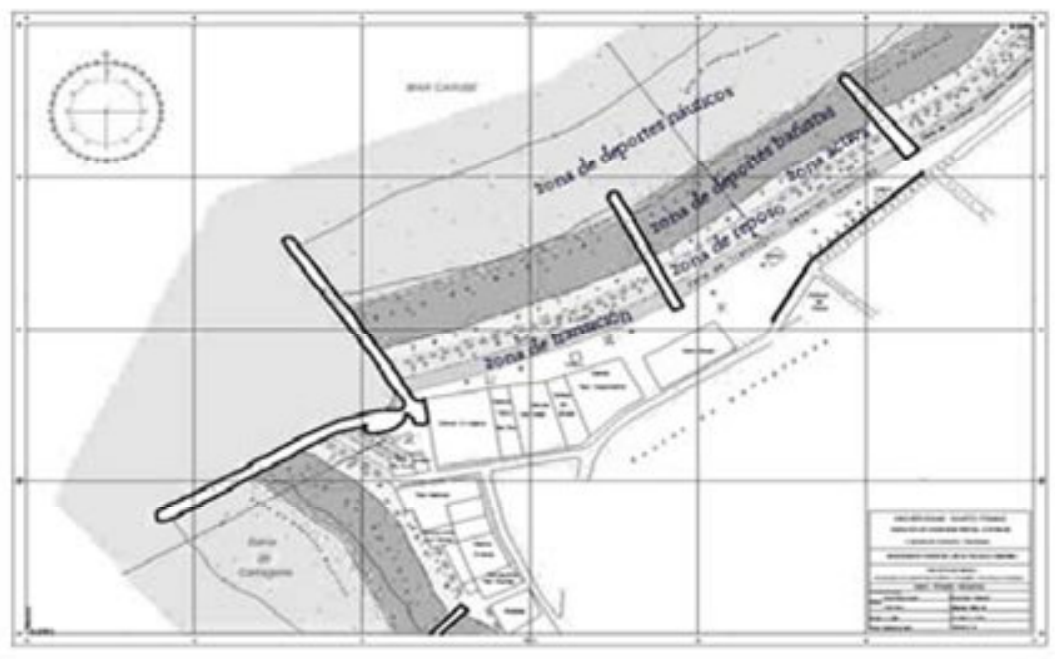

Fuente: Gutiérrez (21) 
PLANO No. 2b. PLAYA SECTOR BOCAGRANDE

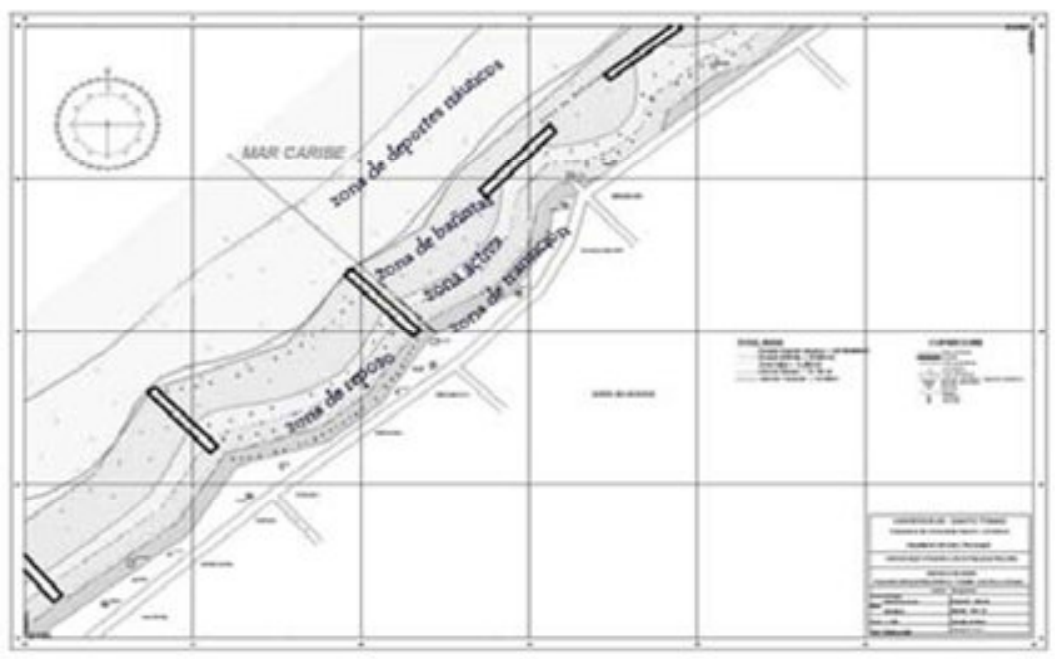

Fuente: Gutiérrez (21)

\section{DATOS}

La información obtenida revela que $54,1 \%$ de los encuestados corresponden al género masculino y $45,9 \%$ al femenino. Mientras el $65,2 \%$ de los hombres presta el servicio de carpas y el $34,8 \%$ se dedica a las ventas en módulo; de las mujeres, el $64,1 \%$ se dedica a la venta de frutas, mientras que el restante $35,9 \%$ vende en módulos. Ambos grupos mantienen una gran responsabilidad dentro de su núcleo familiar en tanto que mayoritariamente son jefes de hogar $(95,7 \%$ de los hombres y $82,1 \%$ de las mujeres) y poseen, principalmente, dos personas a cargo (45\% de los hombres y $40,6 \%$ de las mujeres jefes de hogar). Este hecho cobra aún más importancia si se tiene en cuenta que para el $81,2 \%$ de los encuestados la labor que desempeñan en las playas es su único medio de subsistencia.

El instrumento interrogó sobre los ingresos que reciben los trabajadores estacionarios en las diversas temporadas turísticas en Cartagena, esto permite construir su distribución. Para ello se consideraron tres temporadas: la i) temporada baja que corresponde a los meses de menor flujo de visitantes en las playas, siendo en total de 9 meses; la ii) temporada media se presenta en los meses en el que el flujo de visitantes a las playas aumenta, originado por periodos cortos de vacaciones de colegios y universidades, se presenta en Semana Santa (abril) y en las vacaciones de junio; y la c) temporada alta generada cuando el flujo de visitantes en las playas aumenta de tal magnitud, que se da una ocupación total de todo el espacio público disponible, este periodo se presenta en navidad y a final de año (dura 1 mes). 
La distribución de los ingresos reportados según la temporada turística se presenta en el Cuadro 2. En temporada baja la mayoría de los ingresos que reciben los vendedores están por debajo de los \$300.000, mientras que en temporada media la mayoría se ubica en un rango intermedio, en la temporada alta más de la mitad del ingreso recibido es superior a los $\$ 600.000$. Este incremento del ingreso que se observa en temporada alta sirve para equilibrar, el promedio mensual de los ingresos que se tiene en los demás meses del año. Según el tipo de actividad, la venta de frutas concentra a los individuos que perciben el menor nivel de ingreso $(88 \%$ recibe de $\$ 100.000$ a 300.000), siendo la actividad, en promedio, peor remunerada, en tanto que en las otras dos actividades se registran mayoritariamente ingresos entre $\$ 300.000$ y $\$ 600.000$.

CUADRO No. 2. DISTRIBUCIÓN DE LOS INGRESOS POR TEMPORADA

\begin{tabular}{|c|c|c|c|}
\hline Rango de ingreso & $\begin{array}{c}\text { Ingresos en } \\
\text { temporada baja } \mathbf{9} \\
\text { meses) }\end{array}$ & $\begin{array}{c}\text { Ingresos en } \\
\text { temporada media (2 } \\
\text { meses) }\end{array}$ & $\begin{array}{c}\text { Ingresos en } \\
\text { temporada alta } \\
\text { (1 mes) }\end{array}$ \\
\hline Menos de $\$ 100.000$ & $36,5 \%$ & $14,1 \%$ & $0 \%$ \\
\hline \begin{tabular}{c|c|c|} 
De $\$ 100.000$ a \\
$\$ 300.000$
\end{tabular} & $52,9 \%$ & $49,4 \%$ & $14,1 \%$ \\
\hline \begin{tabular}{c|c|c|} 
De $\$ 300.001$ a \\
$\$ 600.000$
\end{tabular} & $10,6 \%$ & $30,6 \%$ & $23,5 \%$ \\
\hline Más de $\$ 600.000$ & $0 \%$ & $5,9 \%$ & $62,4 \%$ \\
\hline Total & $100 \%$ & $100 \%$ & $100 \%$ \\
\hline
\end{tabular}

Fuente: Cálculo y diseño de los autores

Otro aspecto que se indagó en el cuestionario es el relativo al nivel de inversión que el trabajador estacionario tiene que efectuar para poner en marcha su negocio. La actividad que mayor capital necesita es la venta en módulos, pues el $73,3 \%$ de los individuos que la desempeñan invierten más de \$200.001 mensualmente. El caso contrario se presenta con el servicio de carpas, la inversión que efectúan en promedio el $96 \%$ de sus trabajadores se ubica entre $\$ 50.000$ y $\$ 200.000$.

Se encontró que la rentabilidad que obtienen los prestadores del servicio de carpas en las playas es mayor que el resto de actividades. La mayoría de los individuos que invierten mensualmente sumas entre $\$ 50.000$ y $\$ 100.000$ reportan ingresos entre $\$ 300.000$ y $\$ 600.000$ (76\% de los carperos). La venta de frutas es la que menor rentabilidad genera a los que la desempeñan, en tanto que la inversión media mensual que se hace entre $\$ 100.001$ y $\$ 200.000$ reporta entre $\$ 100.000$ y 300.000 (88\% de los vendedores de frutas). Esta aproximación a la rentabilidad de los negocios posee un carácter meramente aproximativo, que se sugiere profundizar en posteriores estudios al respecto. 
En cuanto a la caracterización del puesto de trabajo la evidencia muestra que $89,4 \%$ de los individuos encuestados manifiestan haber estado establecidos en el mismo sitio de trabajo, siendo en la mayoría de los casos el mismo encuestado el primero en ubicarse en el lugar de trabajo (61,2\%), seguido de la opción otra persona $(17,7 \%)$, algún familiar $(15,3 \%)$ o algún amigo $(5,9 \%)$.

Las apreciaciones de los encuestados sobre la estabilidad que perciben sobre su lugar de trabajo permiten entender que efectivamente éste es estable $(88,2 \%)$, encontrándose distribuidas tales participaciones dentro de las actividades, de una manera prácticamente homogénea. Al contrastar lo anterior con el tiempo (en años) que han estado desempeñándose en tal actividad, se observa una relación positiva entre estas dos variables; particularmente los que tienen 16 o más años de estar en su puesto de trabajo actual concentran el $80 \%$ de las respuestas afirmativas sobre estabilidad. Incluso si se relaciona la primera variable con la consideración de permanecer mucho tiempo más en el mismo sitio de labores, se encuentra una relación semejante, conteniendo el intervalo mencionado alrededor de $77,6 \%$ de las respuestas afirmativas. Además, atendiendo al criterio de relativa seguridad económica que brinda el trabajo que desempeñan en la playa, estos individuos consideran que en caso de no poder volver a trabajar en éste, pretenden legarlo a algún miembro de su familia $(95,3 \%)$, sobre todo cuando entre los demás trabajadores que laboran en el espacio próximo existen buenas o excelentes relaciones $(87 \%)$.

En el módulo de control institucional del cuestionario aplicado, se registran mayoritariamente respuestas que indican un estado positivo de este componente; el 92,9\% de los trabajadores estacionarios encuestados manifestó poseer un carnet que autoriza la utilización del espacio público para fines comerciales, además el $94,1 \%$ de la muestra reporta el uso de un uniforme en el desempeño de su labor. El resultado menos favorable en términos relativos es con relación al aseo del puesto de trabajo, aquí el 29,4\% manifestó que éste se encontraba desaseada.

\section{VARIABLES ASOCIADAS A LA DEMANDA INADECUADA DE PLAYAS POR PARTE DE LOS VENDEDORES ESTACIONARIOS}

En este apartado se analiza la forma de la relación entre diferentes características de los individuos vendedores estacionarios en las playas de Cartagena, y el uso que hacen de la playa en donde desarrollan sus actividades económicas. En el Cuadro No. 3, se muestran las diferentes tablas de contingencia con algunos estadísticos útiles para evaluar la existencia 
de asociaciones entre algunas variables potencialmente explicativas del uso que los vendedores le dan a las playas. Se presentan el coeficiente chi cuadrado, de contingencia, de incertidumbre y la V de Cramér.

Se encontró que el tiempo (años) de laborar en la playa se relaciona moderadamente con el uso que se le da a este espacio público quedando claro que tal asociación no es debida al azar. Se infiere que la consideración del tiempo que una persona lleva de haber establecido su negocio en la playa disminuye la incertidumbre (en un $8,6 \%$ ) sobre los resultados de cualquier ejercicio de predicción sobre el uso adecuado (o no), que no lo incluyera El rango de ingresos recibidos en el negocio efectivamente se relaciona con el uso inadecuado que se haga de las playas. Se puede observar que la mayoría de los individuos trabajadores de los rangos de mayores (menores) ingresos, demandan adecuadamente (inadecuadamente) el espacio público de la playa, es decir, se encuentran dentro (fuera) del área de reposo, que es el espacio asignado para el desarrollo de su labor; la relación directa que se halló (mayor ingreso $\rightarrow$ demanda adecuada) cuando se emplea para predecir a la variable dependiente, se encuentra que es poca la incertidumbre que logra reducir (alrededor del 6\%).

Dividiendo a los trabajadores estacionarios por género, se encontró que la demanda inadecuada de espacio público no es independiente de estas dos categorías, sin embargo a pesar de que ser hombre o mujer determina el uso del espacio playero, la intensidad de esta relación es relativamente baja, y la incertidumbre que se reduciría al tratar de controlar la demanda de espacio púbico por esta variable es poca $(2,8 \%)$, aunque estadísticamente significativa.

Las variables que involucran el reconocimiento o la presencia de la autoridad (DIMAR y Alcaldía de Cartagena), mantienen una relación estadística poco clara con la forma en que se demanda y utiliza el espacio público de las playas (significativas sólo al $20 \%$ en el caso del reconocimiento y al $25 \%$ en el de la presencia). Incluso cuando se pretende establecer si lo anterior mantiene algún efecto a la hora de predecir o de afectar a la demanda inadecuada de espacio público, se encuentra que la incertidumbre que reducen equivale a cerca del $1,7 \%$.

La condición de que el trabajador y su puesto de trabajo hayan sido reubicados, no guardan relación estadística alguna con la demanda inadecuada que se haga del espacio público de la playa, y no es posible asumir que, luego de controlar estadísticamente por la variable reubicación de los trabajadores, se puede tener un mejor uso de la playa. 
La variable que representa las intenciones de legar el puesto de trabajo, así como el hecho de haber sido el primero en explotar económicamente el espacio actualmente ocupados en la playa para asentar el negocio, guardan muy poca asociación con la demanda de inadecuada que se haga del espacio público.

A través del tiempo los individuos desarrollan sentido de pertenencia por aquello que les ha brindado ciertos niveles de utilidad y beneficio, que en el presente caso resulta ser el espacio público de las playas donde han establecidos sus negocios, incluso lo anterior incluso si no es posible ejercer derechos de propiedad en aquel espacio, por el carácter no exclusivo de los bienes públicos. Se procedió entonces a relacionar el tiempo de permanencia del negocio en la playa con algunas variables que pueden servir como aproximativas a la medida de las buenas prácticas.

En el Cuadro No. 4 se muestra que, en términos generales, cuando se está en un rango superior de años de permanencia, la tendencia es a que existan prácticas adecuadas en la playa, entendiendo éstas como usar uniforme (un mecanismo de identidad y reconocimiento), demandar adecuadamente el espacio público de la playa, mantener aseado el lugar de trabajo, reconocer a la autoridad marítima (Alcaldía de Cartagena o DIMAR) $\mathrm{y}$ haber manifestado tener buenas relaciones con los vecinos.

A pesar de la tendencia anteriormente mencionada, la significancia estadística de las variables da a entender que solamente la demanda inadecuada de playa se relaciona con la permanencia en el lugar. Las demás asociaciones del Cuadro 4 son escasamente significativas. 


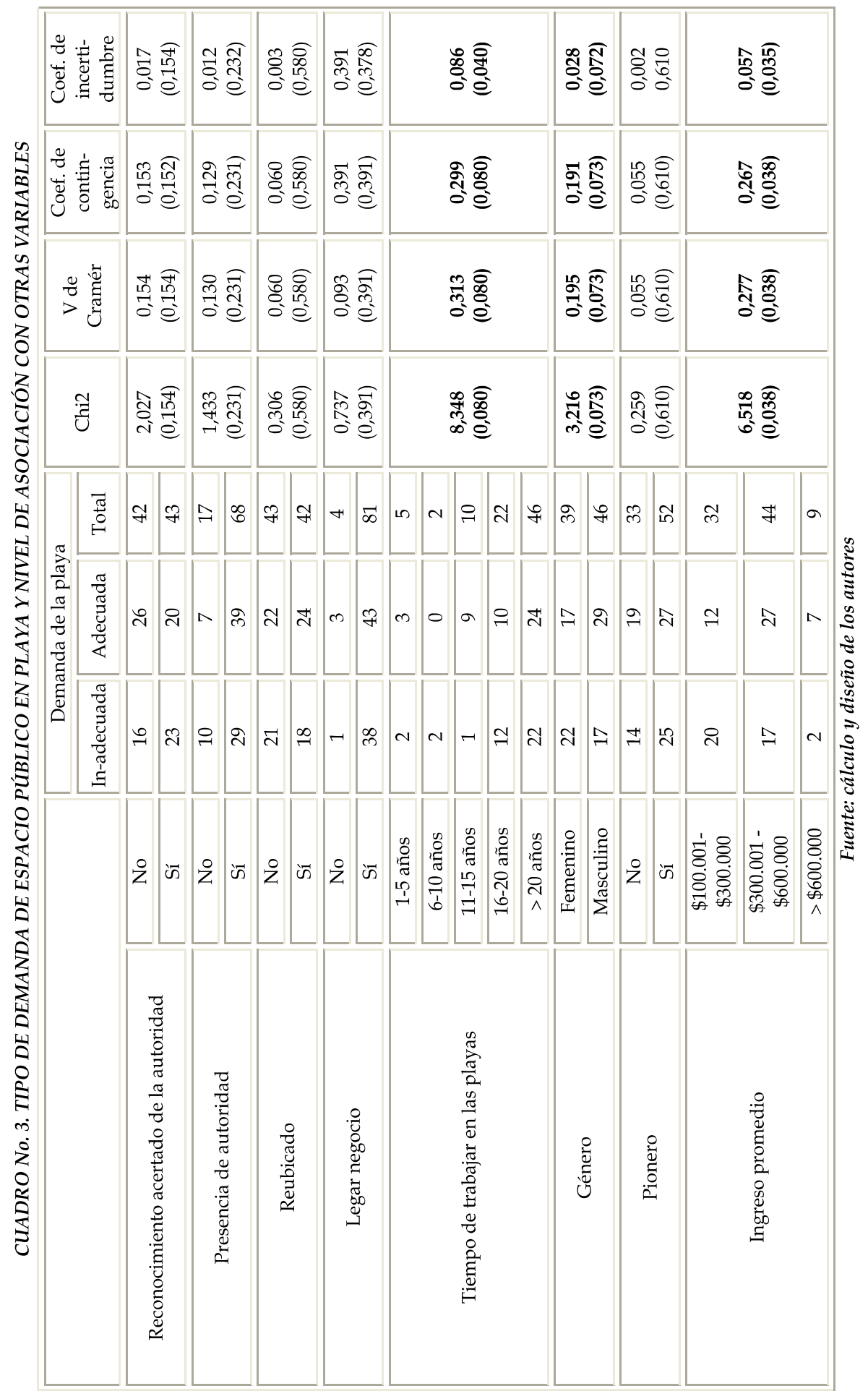




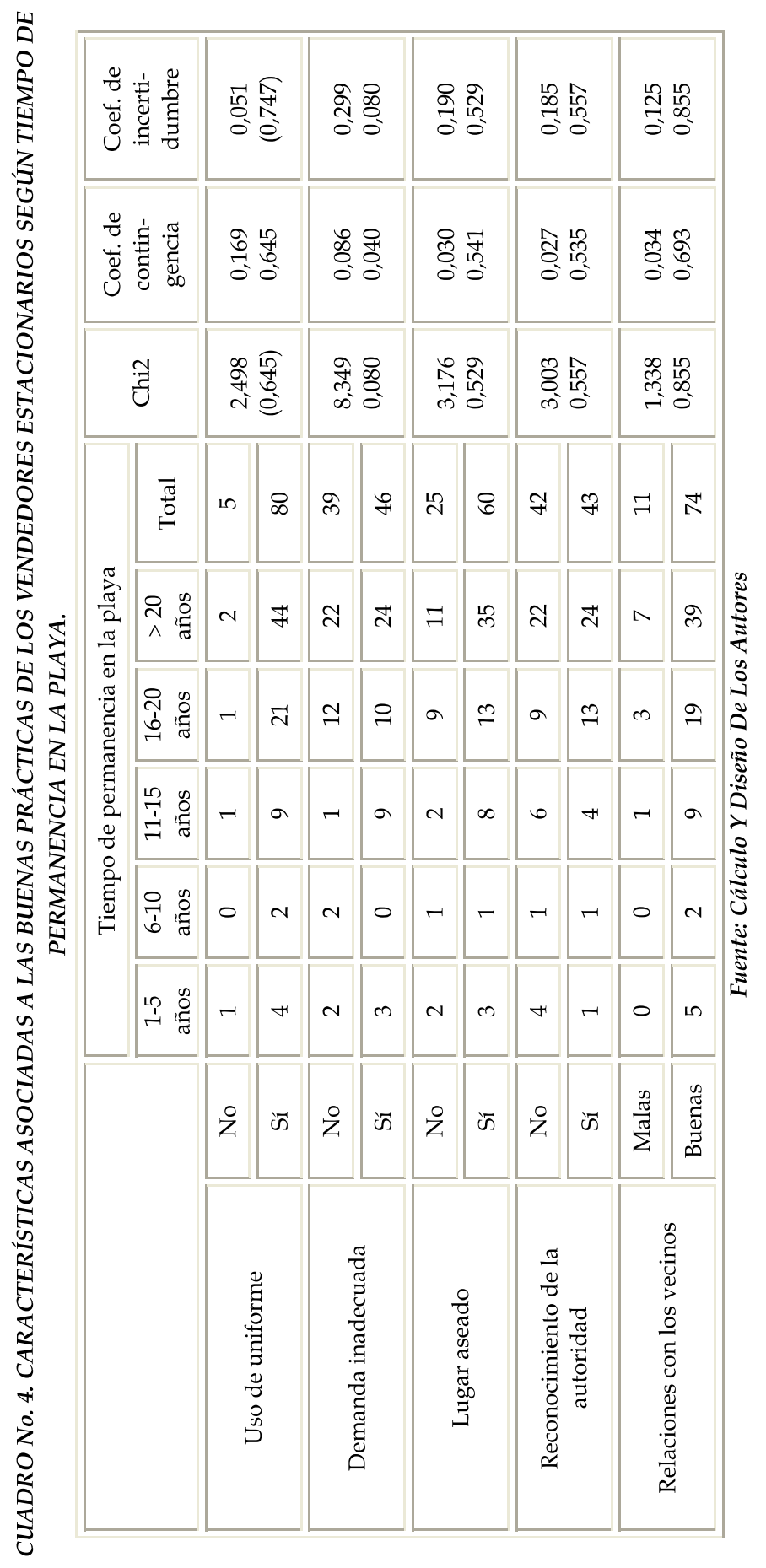




\section{DISCUSIÓN DE RESULTADOS}

La asociación detectada entre nivel de ingreso y demanda inadecuada del espacio público en las playas de Cartagena sugiere que esta conducta es vista como una estrategia para mejorar el ingreso de una población de vendedores que compiten con precios iguales para productos iguales. Bajo estas condiciones de oferta, los compradores en las playas prefieren comprar al vendedor que está más cerca de ellos, es decir su elección optima está determinada por la diferenciación horizontal o espacial (22). Consecuentemente, los vendedores intentan estar en las preferencias de sus compradores, acercándose cada vez más a ellos de forma que termina superando los límites del área destinada para que desarrolle sus funciones, e incurriendo en lo que se ha llamado demanda inadecuada del espacio de las playas

La ausencia de relación estadística entre la condición de reubicado del vendedor con la demanda inadecuada que este haga del espacio público de la playa, resulta llamativa teniendo en cuenta que cerca del $50 \%$ de los encuestados declaró que logró ubicarse en el espacio que actualmente ocupa como consecuencia de un proceso de reubicación (Ver cuadro 5). Otros trabajos han relacionados los procesos de reubicación de vendedores informales con disminución los ingresos de estos (20). Si asociamos esto a lo mencionado en el aparte anterior sería de esperar una relación directa entre reubicación, pérdida de ingresos y tendencia a realizar una demanda inadecuada de la playa en búsqueda de aumentarlos. Sin embargo, el análisis de ingresos de la población de vendedores reubicados en las playas no muestra una tendencia de ingresos diferenciada de la del resto de la población encuestada. En consecuencia no se verifica la relación entre reubicación y obtención de menores ingresos y por lo tanto se descarta la tendencia de esta población a prácticas relacionadas con demanda inadecuada del espacio en las playas. (18)

CUADRO No. 5. ¿CÓMO LOGRÓ UBICARSE?

\begin{tabular}{|ll|r|r|r|r|}
\hline & & & \multicolumn{1}{c|}{$\begin{array}{c}\text { Porcentaje } \\
\text { válido }\end{array}$} & $\begin{array}{r}\text { Porcentaje } \\
\text { acumulado }\end{array}$ \\
\hline Válidos & legado familiar & 13 & 15.3 & 15.3 & 15.3 \\
& reubicado & 42 & 49.4 & 49.4 & 64.7 \\
& iniciativa propia & 30 & 35.3 & 35.3 & 100.0 \\
Total & 85 & 100.0 & 100.0 & \\
\hline
\end{tabular}

Fuente: Diseño de los Autores 
Los vendedores con un mayor número de años de tener negocios en la playa, tienden a desarrollar prácticas relacionadas con una demanda adecuada de esta. Debido a que los controles distritales han estado limitados a la carnetización o exigencia de pertenecía a alguna asociación para conceder permisos de trabajo, se ha reforzado una relación de mercadeo de estos espacios entre la administración distrital y los vendedores. De esta forma, los individuos con un mayor número de años en esta actividad, han encontrado la forma de limitar su competencia al utilizar estos requisitos como una manera de establecer mecanismos de exclusión para potenciales competidores, garantizando así su estabilidad y mayor nivel de ingresos. Este panorama conduce a expresiones de privatización del espacio público como las señaladas por Vasquez Ramirez (19) en el caso de las plazas de la Ciudad de Pereira y el Centro de Bogotá como señala Donovan (18).

\section{CONCLUSIONES}

Los resultados de este estudio sugieren que existe demanda inadecuada de las playas de playas de Cartagena de Indias. Los vendedores que en ellas trabajan, utilizan la diferenciación espacial como una estrategia para generar ventajas frente a sus competidores que ofrecen productos similares a precios similares. De esta forma todos tratan de estar lo más cerca posible de los compradores y terminan por abandonar los espacios inicialmente asignados para su función, generando problemas de aglomeración e invasión de las zonas destinadas a visitantes y bañistas. La falta de claridad institucional para señalar quien tiene la responsabilidad de regular y solucionar este problema, se refleja en la falta de unidad de las respuestas entre los vendedores al pedírseles que identifiquen la autoridad que supervisa y controla sus actividades en la zona de las playas. La ineficiencia institucional para solucionar o controlar el fenómeno es evidente, al punto que aquellos vendedores con mayor números de años explotando económicamente el lugar, han convertido los incipientes mecanismos de control sobre la actividad, en una manera de asegurar su permanencia en el lugar y crear barreras a la entrada de nuevos competidores; anulando así su posible efectividad para orientar la conducta y generación de buenas prácticas en la explotación económica de las playas.

\section{REFERENCIAS BIBLIOGRÁFICAS}

1. Cartagena De Indias. Alcaldía Mayor. Decreto 328 de 1995, 7 de Abril. Cartagena: Alcaldía Mayor; 1995. 
2. Departamento Administrativo Nacional de Estadísticas (DANE). Encuesta de Turismo en Hogares. Colombia. 2003.

3. Ministerio De Comercio, Industria Y Comercio. Viceministerio de Turismo. Colombia. Plan Sectorial De Turismo 2003-2006. Turismo Para Un Nuevo Pais (Preliminar). 2003. [Internet]. Disponible en: http:// www.mincomercio.gov.co/eContent/documentos/turismo/2003/ turismonuevopais/Plansectorialdeturismo/PlanSectorial.doc.

4. Ministerio De Comercio, Industria Y Comercio. Viceministerio de Turismo. Colombia. Plan Sectorial De Turismo 2008-2010. Colombia, Destino Turistico De Clase Mundial. 2008. [Internet]. Disponible en: http://www.mincomercio.gov.co/eContent/Docu mentos/ turismo/2008/PlanSectoria2007-2010-2.pdf.

5. Alcaldía Mayor de Cartagena de Indias, Corporación Turismo Cartagena de Indias, Instituto de Patrimonio y Cultura (IPCC), Corporación Cartagena 2011, Programa de las Naciones Unidas (PNUD). Cartagena de indias es Nuestro Patrimonio. Plan Sectorial de turismo de Cartagena de Indias. 2004. [Internet]. Disponible en: http:/ / www. turismocartagenadeindias.com/image/plansectorial. zip.

6. Baumol W. J., Oates W. E. La Teoría de la Política Económica del Medio Ambiente. Barcelona: Antoni Bosch; 1982.

7. Stiglitz J. E. La Economía del Sector Público. $3^{\mathrm{a}}$ ed. Barcelona: Antoni Bosch; 2000.

8. Hanley N, Shogren J, White Ben. Environmental Economics in Theory and Practice. New York: Oxford University Press; 1997.

9. Hardin G. La Tragedia de los Comunes [traducción]. Gaceta Ecologica. [Internet]. 1995; 37:69. Disponible en: http://www2.ine.gob.mx/ publicaciones/consultaPublicacion.html?id_pub=231.

10. Constitución Política de Colombia 1991. Bogotá D. C. Panamericana Editorial.

11. Código Civil Colombiano. [Internet]. [Consultado 15 de junio de 2005]. Disponible en: http://www.uniderecho.com/leer_ley_Codigos-Colombianos_36_556.html.

12. Régimen Político, Administrativo y Fiscal de los Distritos Portuario e Industrial de Barranquilla, Turístico y Cultural de Cartagena de Indias y Turístico, Cultural e Histórico de Santa Marta. Ley 768/2002 de Julio 31. Diario Oficial No. 44.893, (7-08-2002). [Internet]. Disponible en: http://www.secretariasenado.gov.co/ senado/basedoc/ ley/2002/ley_0768_2002.html.

13. Resolución 438 de 1986. Dirección General Marítima. Bogotá. 1986.

14. Decreto-Ley por el cual se reorganiza la Dirección General Marítima y Portuaria. Decreto-Ley 2324 de 1984. [Internet]. [Consultado 15 de junio de 2005]. Disponible en: http://www.cioh.org.co/ index. 
php?option $=$ com_docman\&task $=$ doc_download\&gid $=277 \& I t e m i d=$.

15. Gordon H. The Economic Theory of a Common-Property Resource: the Fishery. The Journal of Political Economy [Internet] 1954 [consultado diciembre 14 de 2007]; 62 (2):124-142. Disponbile en: http:/ / www.jstor.org/stable/1825571.

16. Hotelling H. Stability in Competition. The Economic Journal [Internet]. [Consultado 7 de noviembre de 2006]; 1929 39(53):41-57. Disponible en: http://www.jstor.org/stable/2224214.

17. Ostrom E. governing the commons: the evolution of institutions for collective action. Cambridge University Press. 1990.

18. Donovan M. Space Wars in Bogotá: The Recovery of Public Space and its Impact on Strett Vendors. [Trabajo de grado para optar por el Master in City Planning]. MIT. Febrero, 2002.

19. Vázquez T. Espacio Público: un Territorio en Disputa. Revista de Ciencias Humanas. 2005; 35: 161-172. Universidad Tecnológica de Pereira. [Internet]. Disponible en: http://www.utp.edu.co/php /revistas/cienciasHumanas/docsFTP/17131espacioCpublico. pdf.

20. Rocha R, Sánchez F. Ventas Callejeras y Espacio Público. Efectos Sobre el Comercio de Bogotá. Documentos Cede. 2006; 12: 1-27. Universidad de los Andes.

21. Gutiérrez C. Recuperación del espacio público, ambiental y paisajístico de las playas de Cartagena. [Tesis de Grado] Universidad Santo Tomas. 2003.

22. Tirole, Jean. The Theory of Industrial Organization. The MIT Press. Seventh Printing. 1994.

\section{BIBLIOGRAFIA RECOMENDADA}

Ley de Reforma Urbana. Ley 9 de 1989. [Internet]. [Consultado 15 de junio de 2005]. Disponible en: http:/ / www.inurbe.gov.co/nuestraCasa / Documentos/ley_9.htm.

Mankiw G. Principios de Economía, Madrid. Harvard University Press, McGraw-Hill. 2002.

Peston M. Bienes Públicos y Sector Público, Madrid. McMillan-VinceVives. 1975.

Field B, Field M. K. Economía Ambiental. 3ª ed. Madrid: McGraw-Hill; 2002.

Nicholson W. Microeconomía Intermedia y Sus Aplicaciones. $8^{\mathrm{a}}$ ed. Bogotá: Mc Graw Hill; 2001. 